\title{
Efetividade profilática e terapêutica do laser de baixa intensidade na mucosite bucal em pacientes submetidos ao tratamento do câncer
}

\author{
Prophylactic and therapeutic effectiveness of low level laser on oral \\ mucositis in patients undergoing cancer treatment
}

Antonio Eugenio Magnabosco Neto*

Fernando Henrique Westphalen

\section{Resumo}

Objetivo: o objetivo desta revisão de literatura é apresentar os diferentes protocolos clínicos randomizados utilizando o laser de baixa intensidade no manejo da mucosite bucal nos últimos 18 anos de estudo. Revisão de literatura: a mucosite bucal persiste como grande problema a ser solucionado na oncologia clínica. Esse efeito citotóxico limita a ingestão alimentar por via bucal, em razão da dor e do desconforto para mastigar e/ ou deglutir, podendo ocorrer agravamento do estado nutricional, contribuindo para aumento da morbidade e tempo de internação hospitalar, proporcionando impacto negativo na qualidade de vida do paciente oncológico e no seu tratamento. Os distúrbios na integridade e função da cavidade bucal devem-se ao fato de que a radioterapia e quimioterapia não são capazes de destruir as células tumorais sem lesionar células normais. Inúmeros tratamentos são descritos na literatura, e diversas são as metodologias para o tratamento da mucosite bucal. Ainda assim, não existe consenso acerca da melhor estratégia para prevenção e terapêutica da enfermidade. Considerações finais: vários estudos têm mostrado bons resultados com o uso do laser de baixa potência, e suas principais indicações incluem ação anti-inflamatória, analgésica e indutora da reparação tecidual. Porém, não existe até o momento um protocolo único definido desse promissor recurso a ser empregado na terapêutica dessa patologia.

Palavras-chave: Laserterapia. Baixa intensidade. Mucosite oral. Quimioterapia. Radioterapia.

\section{Introdução}

Os efeitos do laser de baixa intensidade podem levar a uma aceleração no processo de cicatrização de feridas, os quais são atribuídos ao estímulo de diversos processos metabólicos ${ }^{1}$, mediante a conversão da energia luminosa que ele aporta, transformando, por meio de processos bioquímicos e fotofísicos, a luz em energia útil para a célula ${ }^{2}$.

A conversão fotoquímica é absorvida pelos fotorreceptores celulares, onde a molécula que absorve a luz transfere essa energia para outras moléculas, resultando na ativação celular e em reações químicas no tecido circunvizinho. Essa energia celular disponível, envolvendo as mitocôndrias e demais estruturas celulares, provoca o fenômeno de biomodulação celular ${ }^{3,4}$. Apesar desses efeitos, também ocorre um aumento significativo na síntese de proteínas e DNA, modulação da produção dos fatores de crescimento $^{5}$ por macrófagos, proliferação de queratinócitos, aumento da população e de granulação de mastócitos e angiogênese ${ }^{3}$, apresentando melhor interação com as fibras de colágeno e enzimas específicas, estimulando a microcirculação local e auxiliando o sistema linfático ${ }^{6-8}$. Além de o laser ser bem tolerado pelos pacientes, sua irradiação produz efeitos benéficos, como propriedades anti-inflama- 
tórias, analgésicas e de reparação tecidual ${ }^{7,8}$ durante a abordagem da mucosite bucal. Dessa forma, o comprimento de onda e a dose de energia influenciam significativamente esses resultados ${ }^{8-10}$.

O emprego do laser de baixa potência elimina a dor já na primeira aplicação, promovendo o aumento na concentração de $\beta$-endorfina no líquor cefalorraquidiano, ativando a reparação tecidual, favorecendo a proliferação de fibroblastos e a produção de fibras elásticas e colágenas, elevando, assim, a celularidade dos tecidos irradiados ${ }^{11,12}$.

A fotobiomodulação a laser tem sido utilizada como forma de prevenção e tratamento da mucosite bucal apresentada por pacientes, recebendo altas doses de quimioterapia e/ou radioterapia, e tem obtido respostas positivas do ponto de vista clínico e funcional, podendo ser usada isoladamente ou associada a tratamento medicamentoso, de modo a reduzir sua severidade ${ }^{13-17}$.

Vários estudos clínicos com diferentes protocolos têm sido realizados com intuito de comprovar a eficácia do laser de baixa intensidade no manejo da mucosite bucal ${ }^{18}$. Contudo, parâmetros como período de início e término de sua aplicação, potência do equipamento, comprimento de onda, densidade de energia, área da fibra ótica e frequência são fatores que influenciam no resultado do tratamento, assim como seus efeitos e razões de aplicação tanto curativa como profilática ${ }^{19-26}$.

\section{Materiais e método}

Trata-se de uma revisão sistematizada de literatura, sem meta-análise, compilada a partir da seleção de 24 artigos científicos de periódicos, relatando estudos clínicos randomizados sobre a efetividade profilática e terapêutica do laser de baixa intensidade no manejo da mucosite bucal em pacientes submetidos ao tratamento do câncer, no período compreendido entre 1995 e 2013. Os dados coletados foram organizados em planilha do Microsoft Office Excel para Windows 2007, versão SP3 MSO (12.0), visando a facilitar a identificação e a análise dos aspectos relevantes para o estudo.

\section{Revisão da literatura}

\section{Terapia a laser}

A palavra "laser" é um acrônimo para amplificação de luz por emissão estimulada de radiação. É uma forma de radiação eletromagnética não ionizante, altamente concentrada, que tem capacidade de emitir luz com comprimento de onda único e definitivo, com características distintas ${ }^{27}$.

Quando é absorvida em diferentes tecidos, dependendo do tipo de laser, a radiação resulta em efeitos fotoquímicos, térmicos e não lineares ${ }^{3}$. Essa interação com o tecido está diretamente relacionada com o comprimento de onda, dose ou fluência (densidade de energia), intensidade (densidade de potência), regime de operação do laser, taxa de repetição ou frequência do pulso e características óticas do tecido, como o coeficiente de absorção e espalhamento, para que se possa estabelecer o número de aplicações e dose ótima de radiação $0^{3,22-25,28}$ Os efeitos da energia dos fótons absorvidos pelas células no tecido irradiado, nas terapias com laser de baixa intensidade, são fotoquímicos, fotofísicos e/ou fotobiológicos, excluindo-se os efeitos térmicos, na medida em que essa terapia não promove aquecimento ${ }^{3,26}$. Dessa maneira, auxiliam significativamente a cicatrização e a diminuição da dor ${ }^{27-29}$.

Por ser uma técnica não invasiva, simples e atraumática, sem relatórios clínicos de toxicidade, a aplicação de luz é ideal para o tratamento de tecidos sem risco de danificar as células saudáveis ${ }^{30-43}$

\section{Utilização da terapia a laser de baixa intensidade na prevenção e tratamento da mucosite bucal: estudos clínicos randomizados}

Os lasers de baixa intensidade são utilizados com propósito terapêutico, em virtude das baixas densidades de energia usadas e do comprimento de onda capaz de penetrar nos tecidos ${ }^{7}$. Seu mecanismo de ação na mucosite bucal tem sido proposto por vários autores ${ }^{31}$, aplicado tanto de forma preventi$\mathrm{va}^{7,13,14,18,22,24-27,36,44}$.quanto curativa ${ }^{17,20,27-34,39,44-50}$. De acordo com Karu et $\mathrm{al}^{3}$, os lasers mais frequentemente utilizados para a bioestimulação dos tecidos estão na região do espectro eletromagnético, compreendida entre 630 e $1000 \mathrm{~nm}$, o que abrage a região do vermelho e infravermelho próximo.

Contudo, segundo Basford ${ }^{37}$, para obter-se o efeito da bioestimulação, é necessária uma densidade de energia entre 1 a $4 \mathrm{~J} / \mathrm{cm}^{2}$ e uma potência de 10 a $90 \mathrm{~mW}$. Lubart et al..$^{50}$ observaram que, em relação ao comprimento de onda a partir de $540 \mathrm{~nm}$ e entre $600 \mathrm{~nm}$ a $900 \mathrm{~nm}$, a mitose das células é significativamente acelerada, aumentando a velocidade de produção do colágeno e dos fibroblastos ${ }^{2,47,49}$.

Barasch et al.$^{13}$ realizaram um estudo controlado, prospectivo e duplo-cego, envolvendo 20 pacientes submetidos a transplante de medula óssea (TMO), utilizando um laser de $\mathrm{HeNe}(632,8 \mathrm{~nm}, 25$ $\mathrm{mW}$ e $1,0 \mathrm{~J} / \mathrm{cm}^{2}$ ) preventivo do $\mathrm{D}-1$ até o $\mathrm{D}+3$, unilateralmente, nas regiões de mucosa jugal, mucosa labial, borda e ventre lingual, assoalho bucal e palato. Mantendo o lado oposto como grupo controle, mostraram que a terapia com laser foi bem tolerada pelos pacientes e sua aplicação, responsável pela redução da severidade da mucosite bucal. 
Em estudo duplo-cego, randomizado, envolvendo 30 pacientes que receberam tratamento quimioterápico previamente ao transplante de medula, Cowen et al. ${ }^{7}$ utilizaram, para fins preventivos, o laser $\mathrm{HeNe}(632,8 \mathrm{~nm}, 60 \mathrm{~mW}$, e 1,5 J/cm²), diariamente por cinco dias consecutivos (D -5 ao D -1). Verificaram que a aplicação diária do laser reduziu a intensidade, a severidade e a duração da mucosite, além da dor e da necessidade de administração de morfina. Em estudo prospectivo randomizado, duplo-cego, Bensadoun et al. ${ }^{4}$ relacionaram a prevenção de mucosite com o emprego do laser de baixa intensidade, em pacientes tratados com radioterapia em cabeça e pescoço. Os autores concluíram que irradiações com laser de baixa intensidade são capazes de reduzir a severidade e duração da mucosite bucal associada com a radioterapia.

Migliorati et al. ${ }^{32}$, realizando estudo piloto em que avaliaram a utilização do laser no controle da dor associada à mucosite bucal após transplante de medula óssea e alta dose de quimioterapia para mieloablação, verificaram que o laser teve papel importante nesse processo.

Em estudo randomizado duplo-cego, desenvolvido para determinar aplicações preventivas do laser de $\mathrm{HeNe}\left(632,8 \mathrm{~nm}, 60 \mathrm{~mW}\right.$ e $2 \mathrm{~J} / \mathrm{cm}^{2}$, Bensadoun e Ciai $^{30}$ observaram que a aplicação de laser atrasou o início da mucosite, atenuou seu pico de severidade e reduziu sua duração.

Sandoval et al. ${ }^{15}$ avaliaram 18 pacientes com idade entre 4 e 82 anos, recebendo aplicações diárias de laser $\left(630 \mathrm{~nm}, 30 \mathrm{~mW}, 2 \mathrm{~J} / \mathrm{cm}^{2}\right)$, até que os sintomas da mucosite cessassem. Os autores concluíram que o laser de baixa potência foi bem tolerado e demonstrou efeitos benéficos no manejo da MO. Nes e Posso ${ }^{17}$ investigaram os efeitos clínicos da terapia a laser, utilizaram um meio ativo AsGa$\mathrm{Al}\left(830 \mathrm{~nm}, 250 \mathrm{~mW}, 35 \mathrm{~J} / \mathrm{cm}^{2}\right)$ por ponto, durante cinco dias consecutivos. Os resultados demonstraram uma redução, estatisticamente significante, de $67 \%$ da sintomatologia dolorosa relatada pelos pacientes.

Em estudo randomizado e controlado, Maiya et al. ${ }^{18}$ utilizaram laser de $\mathrm{HeNe}(632,8 \mathrm{~nm}, 10 \mathrm{~mW}, 1,8$ $\mathrm{J} / \mathrm{cm}^{2}$ ), preventivamente, em 50 pacientes recebendo radioterapia para carcinoma na cavidade bucal. Os autores constataram que o laser atrasou o início do desenvolvimento e atenuou o pico da mucosite.

Antunes et al. ${ }^{14}$ promoveram estudo randomizado, cego e controlado em 38 pacientes submetidos a TMO, que receberam tratamento com laser de baixa potência (TLBP) InGaAlP $(660 \mathrm{~nm}, 50 \mathrm{~mW}$ e $4 \mathrm{~J} / \mathrm{cm}^{2}$ ), diariamente, do D-7 até a recuperação medular. Doze pacientes apresentaram úlceras na cavidade bucal MO graus 0 e 1, 6 pacientes desenvolveram MO grau 2 e somente 1 paciente desenvolveu MO grau 4, contrapondo-se a 13 pacientes do grupo controle, que desenvolveram MO graus $3 \mathrm{e}$ 4, 4 pacientes com grau 2 e 2 pacientes com grau 1 .

Em estudo randomizado duplo-cego e controlado, para prevenção de mucosite em 70 pacientes submetidos a TMO, Schubert et al. ${ }^{35}$ compararam dois lasers de GaAlAs com diferentes níveis de intensidade, $650 \mathrm{~nm}(40 \mathrm{~mW})$ e $780 \mathrm{~nm}(60 \mathrm{~mW}) \mathrm{na}$ densidade de $2 \mathrm{~J} / \mathrm{cm}^{2}$, preventivo do $\mathrm{D}-7$ ao $\mathrm{D}+2$. Segundo os autores, o laser de $650 \mathrm{~nm}$ obteve melhor desempenho que o de $780 \mathrm{~nm}$, diminuindo a gravidade e a dor da mucosite.

Jaguar et al. ${ }^{27}$ (2007) promoveram estudo comparativo entre dois grupos de pacientes submetidos ao transplante de medula óssea: 24 pacientes receberam aplicação profilática da TLBP e 25 pacientes eram do grupo placebo. Nesse estudo, $40 \%$ da amostra apresentaram algum grau de mucosite, e os escores observados para o grupo controle foram menores no dia 8 .

Abramoff et al. ${ }^{34}$ avaliaram, em estudo clínico prospectivo, randomizado, controlado com placebo, a eficácia e a viabilidade do uso do laser de baixa potência na prevenção e no tratamento da MO quimioinduzida em pacientes jovens. Os autores mostraram que, apesar da pequena amostra utilizada, a maioria dos pacientes (73\%) não desenvolveu mucosite no grupo profilático e houve recuperação rápida no grupo tratado, concluindo que o laser de baixa intensidade utilizado apresentou efeito preventivo e terapêutico.

Arora et al. ${ }^{24}$, em estudo controlado, utilizaram a TLBP de $\mathrm{HeNe}\left(632,8 \mathrm{~nm}, 10 \mathrm{~mW}, 1,8 \mathrm{~J} / \mathrm{cm}^{2}\right)$ como técnica de prevenção de MO. Os autores concluem que a terapia com laser aplicada profilaticamente durante a radioterapia pode reduzir a gravidade da mucosite bucal, a severidade da dor e a incapacidade funcional.

Em ensaio randomizado, controlado por placebo, Kuhn et al ${ }^{31}$ utilizaram a TLBP em 21 crianças e adolescentes com câncer recebendo quimioterapia ou transplante de células-tronco hematopoiéticas (TCTH) logo que desenvolveram mucosite bucal. O estudo mostrou evidências de que a terapia a laser pode diminuir a duração da mucosite bucal quimioinduzida.

Zanin et al. ${ }^{40}$ avaliaram quantitativa e qualitativamente $o$ efeito do laser de diodo na prevenção e no tratamento da mucosite oral em 72 pacientes com câncer de cabeça e pescoço submetidos a radioterapia e quimioterapia. Os autores concluíram que a terapia com laser foi eficaz, melhorando a qualidade de vida do paciente.

Em estudo duplo-cego, randomizado, Djavid et al. ${ }^{39}$ avaliaram o efeito preventivo do laser de baixa intensidade em pacientes recebendo quimioterapia em neoplasias hematológicas. Os resultados mostraram que a incidência da MO para o grupo laser foi de $31 \%$ e para o grupo placebo, de $41 \%$, obtendo 
duração média de cura de 4,8 e 12 dias para ambos os grupos.

Silva et al. ${ }^{46}$ investigaram os efeitos clínicos da terapia com laser de baixa intensidade na prevenção da mucosite bucal em pacientes submetidos ao TCTH. Segundo os autores, os resultados indicaram que o uso preventivo de LBI em pacientes submetidos ao transplante é um instrumento poderoso na redução da incidência de MO.

Em estudo randomizado duplo-cego e controlado, para prevenção e tratamento de mucosite bucal em 70 pacientes com neoplasias malignas da cavidade oral ou orofaringe submetidos à radioterapia, Carvalho et al. ${ }^{48}$ avaliaram a eficácia do tratamento com laser de baixa potência. Os autores concluíram que a terapia com laser de baixa potência, durante a radioterapia, foi eficaz na diminuição da mucosite e no controle da intensidade da dor.

Cauwels e Martens ${ }^{49}$ incluíram 16 crianças com idade média de 9,4 anos em estudo utilizando laser de diodo GaAlAs (830 nm, $150 \mathrm{~mW}$ ). Os autores constataram, após 12 aplicações, que os baixos graus da mucosite bucal obtidos estariam diretamente relacionadas à utilização de menor energia de radiação.

Em estudo triplo cego, randomizado e controlado, Gautam et al. ${ }^{45}$ utilizaram laser de baixa intensidade para a prevenção e o tratamento de mucosite bucal em 221 pacientes com câncer de cabeça e pescoço submetidos a quimiorradioterapia. Os resultados mostraram que houve redução significativa na incidência da mucosite bucal grave, dor associada, disfagia e utilização de analgésicos opiáceos para esses pacientes tratados com laser.

Gouvêa de Lima et al. ${ }^{47}$ avaliaram a eficácia do laser de baixa intensidade na prevenção da mucosite bucal grave em pacientes com câncer de cavidade bucal recebendo quimio e radioterapia concomitantemente. Nesse caso, a terapia com laser de baixa intensidade não foi eficaz na redução da mucosite bucal.

Arbabi-Kalati et al. ${ }^{42}$ observaram o efeito do laser de baixa potência na prevenção da mucosite, xerostomia e dor como resultado da quimioterapia. Os resultados mostraram que o laser de baixa potência foi capaz de diminuir os efeitos deletérios da quimioterapia na cavidade bucal.

Em estudo randomizado, cego, Gautam et al. ${ }^{43}$ avaliaram 220 pacientes. Houve redução significa- tiva ( $p<0,001$ ), na incidência de MO grave, da necessidade de analgésicos opioides.

\section{Resultados}

Os resultados da pesquisa clínica abordando o uso da terapia com laser de baixa potência na prevenção e no tratamento da mucosite bucal rádio e/ ou quimioinduzida têm sido positivos. No entanto, os parâmetros utilizados foram extremamente variados. Período de início e término da aplicação do laser, potência do equipamento, comprimento de onda, densidade de energia e frequência da aplicação são fatores que influenciam no resultado do tratamento, o que pode explicar diferentes variantes encontrados nos artigos selecionados.

A TLBP mostrou-se efetiva quando utilizada com propósito preventivo e destaca-se como alternativa eficaz e viável na redução da gravidade, duração e dor causada pela mucosite bucal. Os efeitos da laserterapia com finalidade preventiva na incidência da mucosite bucal, evidenciados nos artigos pesquisados de acordo com os tipos de tratamentos oncológicos, parâmetros da terapia com laser e forma de avaliação, são verificados na Tabela 1 . 
Tabela 1- Distribuição dos protocolos clínicos aplicados com laser de baixa potência para prevenção da mucosite bucal em pacientes submetidos a terapia antineoplásica

\begin{tabular}{|c|c|c|c|c|c|c|c|}
\hline \multirow[b]{2}{*}{$\begin{array}{l}\text { Referência } \\
\text { (autor/ano) }\end{array}$} & \multirow[b]{2}{*}{ Paciente } & \multirow[b]{2}{*}{$\begin{array}{l}\text { Modalidade } \\
\text { de tratamento }\end{array}$} & \multicolumn{3}{|c|}{ Protocolo laserterapia } & \multirow{2}{*}{$\begin{array}{c}\text { Tipo de } \\
\text { aplicação } \\
\text { (Intra } \\
\text { /extra oral) }\end{array}$} & \multirow[b]{2}{*}{$\begin{array}{c}\text { Tipo de análise } \\
\text { - } \\
\text { MO Index }\end{array}$} \\
\hline & & & Laser & $\begin{array}{l}\text { Comp. } \\
\text { onda } \\
(\mathrm{nm})\end{array}$ & $\begin{array}{c}\text { potência/ } \\
\text { intensidade de } \\
\text { energia/tempo }\end{array}$ & & \\
\hline $\begin{array}{l}\text { Barasch et al. }{ }^{13} \\
(1995)\end{array}$ & Adulto & TCTH c/s ICT & $\mathrm{HeNe}$ & 632.8 & $\begin{array}{c}25 \mathrm{~mW} ; \\
1 \mathrm{~J} / \mathrm{cm}^{2}, 40 \mathrm{seg} .\end{array}$ & $\mathrm{IO}$ & 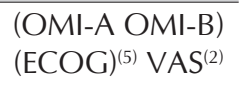 \\
\hline $\begin{array}{l}\text { Cowen et al. } \\
(1997)\end{array}$ & Adulto & TCTH c/ ICT & $\mathrm{HeNe}$ & 632.8 & $\begin{array}{c}60 \mathrm{~mW} \\
1.5 \mathrm{~J} / \mathrm{cm}^{2}, 10 \mathrm{seg} .\end{array}$ & 10 & $\begin{array}{c}\text { Escala de Walsh } \\
\text { et al. }\end{array}$ \\
\hline $\begin{array}{l}\text { Bensadoun et al. }{ }^{4} \\
\text { (1999) }\end{array}$ & Adulto & $\mathrm{RT}-\mathrm{C}$ e $\mathrm{P}$ & $\mathrm{HeNe}$ & 632.8 & $\begin{array}{c}60 \mathrm{~mW} ; \\
2 \mathrm{~J} / \mathrm{cm}^{2}, 23 \mathrm{seg} .\end{array}$ & 10 & $\mathrm{WHO}^{(3)} \mathrm{VAS}^{(2)}$ \\
\hline $\begin{array}{l}\text { Bensadoun e Ciais }{ }^{30} \\
(2002)\end{array}$ & Adulto & RT - C e P & $\mathrm{HeNe}$ & 632.8 & $\begin{array}{c}60 \mathrm{~mW} ; \\
2 \mathrm{~J} / \mathrm{cm}^{2}, 33 \mathrm{seg} .\end{array}$ & 10 & $\mathrm{WHO}^{(3)} \mathrm{VAS}^{(2)}$ \\
\hline $\begin{array}{l}\text { Maya et al. }{ }^{18} \\
(2006)\end{array}$ & Adulto & $\mathrm{RT}-\mathrm{C}$ e $\mathrm{P}$ & $\mathrm{HeNe}$ & 632.8 & $\begin{array}{c}10 \mathrm{~mW} ; \\
1.8 \mathrm{~J} / \mathrm{cm}^{2}, 3 \mathrm{~min} .\end{array}$ & 10 & $\mathrm{WHO}^{(3)} \mathrm{VAS}^{(2)}$ \\
\hline $\begin{array}{l}\text { Antunes et al. }{ }^{14} \\
(2007)\end{array}$ & Adulto & TCTH c/s ICT & InGaAIP & 660 & $\begin{array}{c}50 \mathrm{~mW} \\
4 \mathrm{~J} / \mathrm{cm}^{2}, 16.7 \mathrm{seg} .\end{array}$ & $\mathrm{IO}$ & $\begin{array}{l}\mathrm{OMAS}^{(4)} \\
\mathrm{WHO}^{(3)}\end{array}$ \\
\hline $\begin{array}{l}\text { Schubert et al. }{ }^{35} \\
(2007)\end{array}$ & Adulto & TCTH c/s ICT & GaAlAs & $\begin{array}{c}650 / \\
780\end{array}$ & $\begin{array}{c}40 / 60 \mathrm{~mW} ; \\
2 \mathrm{~J} / \mathrm{cm}^{2}, \mathrm{NE}^{(1)}\end{array}$ & $1 \mathrm{O}$ & $\mathrm{WHO}^{(3)} \mathrm{VAS}^{(2)}$ \\
\hline $\begin{array}{l}\text { Jaguar et al. }{ }^{27} \\
(2007)\end{array}$ & Adulto & TCTH c/s ICT & GaAlAs & 660 & $\begin{array}{c}10 \mathrm{~mW} \\
2.5 \mathrm{~J} / \mathrm{cm}^{2}, 10 \mathrm{seg} .\end{array}$ & 10 & $\mathrm{WHO}^{(3)}$ \\
\hline $\begin{array}{l}\text { Cruz et al. }{ }^{33} \\
(2007)\end{array}$ & Pediátrico & $\mathrm{TCTH} \& \mathrm{Q}$ & GaAlAs & 780 & $\begin{array}{c}60 \mathrm{~mW} ; \\
4 \mathrm{~J} / \mathrm{cm}^{2}, \mathrm{NE}^{(1)}\end{array}$ & 10 & $\mathrm{WHO}^{(3)}$ \\
\hline $\begin{array}{l}\text { Arora et al. }{ }^{24} \\
(2008)\end{array}$ & Adulto & $\mathrm{RT}-\mathrm{C}$ e $\mathrm{P}$ & $\mathrm{HeNe}$ & 632.8 & $\begin{array}{c}10 \mathrm{~mW} ; \\
1.8 \mathrm{~J} / \mathrm{cm}^{2}, \mathrm{NE}^{(1)}\end{array}$ & $1 \mathrm{O} \& \mathrm{EO}$ & $\mathrm{RTOG}^{(7)}$ \\
\hline $\begin{array}{l}\text { Zanin et al. }{ }^{40} \\
(2010)\end{array}$ & Adulto & Q\&RT -C e P & InGaAIP & 660 & $\begin{array}{c}30 \mathrm{~mW} \\
2 \mathrm{~J} / \mathrm{cm}^{2}, \mathrm{NE}^{(1)}\end{array}$ & $\mathrm{IO}$ & $\mathrm{NE}^{(1)}$ \\
\hline $\begin{array}{l}\text { Djavid et al. }{ }^{39} \\
(2011)\end{array}$ & Adulto & Q & GaAlAs & 630 & $\begin{array}{c}30 \mathrm{~mW} ; \\
5 \mathrm{~J} / \mathrm{cm}^{2}, \mathrm{NE}^{(1)}\end{array}$ & 10 & $\mathrm{WHO}^{(3)}$ \\
\hline $\begin{array}{l}\text { Silva et al. }{ }^{46} \\
(2011)\end{array}$ & Adulto & $\begin{array}{c}\text { Q/Q- TCTH c/ } \\
\text { ICT }\end{array}$ & AlGalnP & 660 & $\begin{array}{c}40 \mathrm{~mW} ; \\
4 \mathrm{~J} / \mathrm{cm}^{2}, \mathrm{NE}^{(1)}\end{array}$ & 10 & $\mathrm{WHO}^{(3)}$ \\
\hline $\begin{array}{l}\text { Gouvêa de Lima et } \\
\text { al. }^{47}(2012)\end{array}$ & Adulto & Q\&RT-C e P & GaAlAs & 660 & $\begin{array}{c}10 \mathrm{~mW} ; \\
2,5 \mathrm{~J} / \mathrm{cm}^{2}, 10 \mathrm{seg} .\end{array}$ & 10 & $\mathrm{INC}^{(6)}$ \\
\hline $\begin{array}{l}\text { Gautam et al. }{ }^{45} \\
(2012)\end{array}$ & Adulto & Q\&RT- C e P & $\mathrm{HeNe}$ & 632.8 & $\begin{array}{c}24 \mathrm{~mW} ; \\
3 \mathrm{~J} / \mathrm{cm}^{2}, 125 \mathrm{seg} .\end{array}$ & $\mathrm{IO}$ & $(\mathrm{OMWQ}-\mathrm{HN})^{(8)}$ \\
\hline $\begin{array}{l}\text { Gautam et al. }{ }^{43} \\
(2013)\end{array}$ & Adulto & Q\&RT-C e P & $\mathrm{HeNe}$ & 632.8 & $\begin{array}{c}24 \mathrm{~mW} \\
3 \mathrm{~J} / \mathrm{cm}^{2}, 125 \mathrm{seg} .\end{array}$ & 10 & $(\mathrm{OMWQ}-\mathrm{HN})^{(8)}$ \\
\hline $\begin{array}{l}\text { Arbabi-Kalati et al. }{ }^{4} \\
(2013)\end{array}$ & Adulto & $Q$ & $\mathrm{NE}^{(1)}$ & 630 & $\begin{array}{c}30 \mathrm{~mW} ; \\
5 \mathrm{~J} / \mathrm{cm}^{2}, \mathrm{NE}^{(1)}\end{array}$ & 10 & $\mathrm{WHO}^{(3)} \mathrm{VAS}^{(2)}$ \\
\hline
\end{tabular}

${ }^{(1)} \mathrm{NE}$ : não especificado; (2)VAS: visual analogue scale; (3)WHO: World Health Organization; (4)OMAS: Oral Mucositis Assessment Scale; (5)OMI-A and OMI-B Oral/ ECOG: Oral Mucositis Index Scale and the Eastern Cooperative Oncology Group; (6)INC: Instituto Nacional de Câncer; ${ }^{(7)}$ RTGO: Radiation Therapy Oncology Group; ${ }^{(8)}(\mathrm{OMWQ}-\mathrm{HN})$ : Oral Mucosite Weekly Questionnaire - Cabeça e Pescoço.

TCTH: transplante de células-tronco hematopoiéticas; c: com; s: sem; ICT: irradiação corpórea total; IO: intraoral; EO: extraoral; RT: radioterapia; C e P: cabeça e pescoço; Q: quimioterapia.

Apesar das diferentes metodologias empregadas nos estudos citados utilizando o laser de baixa potência, observou-se redução da morbidade nos pacientes tratados quando comparados com os pacientes controles (simulação) com consequente melhora da qualidade de vida em todos os resultados. Os estudos revelaram que a terapia com o uso do laser profilático parece ser mais eficaz que a abordagem curativa. Porém, todos os resultados apresentaram redução da prevalência, severidade e alívio da dor em diferentes parâmetros empregados, independentemente dos tipos de tratamentos oncológicos mencionados. 
Na Tabela 2, são apresentados os protocolos dos estudos selecionados utilizando o laser de baixa potência com finalidade terapêutica da mucosite bucal em pacientes submetidos ao tratamento do câncer de acordo com os tipos de tratamentos oncológicos, parâmetros da terapia com laser e forma de avaliação.

Tabela 2- Distribuição dos protocolos clínicos aplicados com laser de baixa potência para tratamento da mucosite bucal em pacientes submetidos a terapia antineoplásica

\begin{tabular}{|c|c|c|c|c|c|c|c|}
\hline \multirow{2}{*}{$\begin{array}{l}\text { Referência } \\
\text { (autor/ano) }\end{array}$} & \multirow[b]{2}{*}{ Paciente } & \multirow{2}{*}{$\begin{array}{l}\text { Modalidade de } \\
\text { tratamento }\end{array}$} & \multicolumn{3}{|c|}{ Protocolo laserterapia } & \multirow{2}{*}{$\begin{array}{c}\text { Tipo de } \\
\text { aplicação } \\
\text { (Intra } \\
\text { /extraoral) }\end{array}$} & \multirow{2}{*}{$\begin{array}{c}\text { Tipo de } \\
\text { análise - } \\
\text { MO Index }\end{array}$} \\
\hline & & & Laser & $\begin{array}{c}\text { Comp. } \\
\text { onda(nm) }\end{array}$ & $\begin{array}{c}\text { potência/ } \\
\text { intensidade de } \\
\text { energia/tempo }\end{array}$ & & \\
\hline $\begin{array}{l}\text { Migliorati et al. }{ }^{32} \\
(2001)\end{array}$ & $\begin{array}{c}\text { Adulto/ } \\
\text { Pediátrico }\end{array}$ & $\begin{array}{c}\mathrm{TCTH} \mathrm{c/s} \mathrm{ICT} \\
\& Q\end{array}$ & GaAlAs & 780 & $\begin{array}{c}60 \mathrm{~mW} \\
2 \mathrm{~J} / \mathrm{cm}^{2}, 35 \mathrm{~min} .\end{array}$ & $\mathrm{IO}$ & $\begin{array}{l}\mathrm{WHO}^{(3)} \\
\mathrm{VAS}^{(2)}\end{array}$ \\
\hline $\begin{array}{l}\text { Sandoval et al. }{ }^{15} \\
(2003)\end{array}$ & $\begin{array}{c}\text { Adulto/ } \\
\text { Pediátrico }\end{array}$ & Q\&RT -C e P & $\mathrm{NE}^{*}$ & 660 & $\begin{array}{c}30 \mathrm{~mW} ; \\
2 \mathrm{~J} / \mathrm{cm}^{2}, 66 \mathrm{seg} .\end{array}$ & $\mathrm{IO}$ & $\mathrm{INC}^{(4)}$ \\
\hline $\begin{array}{l}\text { Nes e Posso }{ }^{17} \\
(2005)\end{array}$ & Adulto & Q & AsGaAl & 830 & $\begin{array}{c}250 \mathrm{~mW} \\
35 \mathrm{~J} / \mathrm{cm}^{2}, \mathrm{NE}^{(1)}\end{array}$ & $\mathrm{IO}$ & $\mathrm{VAS}^{(2)}$ \\
\hline $\begin{array}{l}\text { Kuhn et al. }{ }^{31} \\
(2009)\end{array}$ & Pediátrico & $\mathrm{TCTH} \& \mathrm{Q}$ & GaAlAs & 830 & $\begin{array}{c}100 \mathrm{mw} ; \\
4 \mathrm{~J} / \mathrm{cm}^{2}, \mathrm{NE}^{(1)}\end{array}$ & $\mathrm{IO}$ & $\mathrm{INC}^{(4)}$ \\
\hline $\begin{array}{l}\text { Abramoff et al. }{ }^{34} \\
(2008)\end{array}$ & $\begin{array}{c}\text { Adulto/ } \\
\text { Pediátrico }\end{array}$ & Q & GaAlAs & 685 & $\begin{array}{c}35 \mathrm{~mW} \\
2 \mathrm{~J} / / \mathrm{cm}^{2}, 54 \mathrm{seg} .\end{array}$ & $\mathrm{IO}$ & $\mathrm{VAS}^{(2)}$ \\
\hline $\begin{array}{l}\text { Cauwels e } \\
\text { Martens }^{49}(2011)\end{array}$ & Pediátrico & Q & GaAlAs & 830 & $150 \mathrm{~mW} ;{ }^{* * * * *}$ & $\mathrm{IO}$ & $\mathrm{WHO}^{(3)}$ \\
\hline $\begin{array}{l}\text { Carvalho et al. }{ }^{48} \\
(2011)\end{array}$ & Adulto & RT-C e P & $\mathrm{NE}^{(1)}$ & $\begin{array}{l}660 \\
660\end{array}$ & $\begin{array}{c}15 \mathrm{~mW}, 3.8 \mathrm{~J} / \mathrm{cm}^{2} \\
5 \mathrm{~mW}, 1.3 \mathrm{~J} / \mathrm{cm}^{2}\end{array}$ & $\mathrm{IO}$ & $\begin{array}{l}\mathrm{WHO}^{(3)} \\
\mathrm{INC}^{(4)}\end{array}$ \\
\hline
\end{tabular}

${ }^{(1)} \mathrm{NE}$ : não especificado; (2)VAS: visual analogue scale; ${ }^{(3)} \mathrm{WHO}$ : World Health Organization; (4)INC: Instituto Nacional de Câncer; ${ }^{(7)}$ Radiation Therapy Oncology Group.

TCTH: transplante de células-tronco hematopoiéticas; c: com; s: sem; ICT: irradiação corpórea total; IO: intraoral; RT: radioterapia; C e P: cabeça e pescoço; Q: quimioterapia; ${ }^{*+* *}$ densidade de energia adaptada de acordo com a gravidade das lesões de MO.

Os estudos apresentaram grande variedade de dispositivos de laser, variação de protocolos individuais e configurações utilizadas, como parâmetros de comprimento de onda (em nm), densidade de potência ou irradiância $\left(\mathrm{J} / \mathrm{cm}^{2}\right)$ e potência $(\mathrm{mW})$. No entanto, não foram observadas diferenças signifi- cativas na maioria dos ensaios clínicos encontrados nos artigos pesquisados. Na Tabela 3, são apresentados os parâmetros de dosimetria de acordo com os tipos de tratamentos oncológicos, utilizados com propósito preventivo e terapêutico.

Tabela 3 - Distribuição dos parâmetros dosimétricos aplicados com laser de baixa potência dos artigos pesquisados com relação ao tipo de tratamento antineoplásico

\begin{tabular}{c|c|c|c}
\hline \multirow{2}{*}{ Tipo de tratamento } & \multicolumn{2}{|c}{ Parâmetros dosimétricos } \\
\cline { 2 - 3 } TCTH & Comprimento de onda & Densidade de energia \\
RT - C e P & $632.8 \mathrm{~nm}$ a $830 \mathrm{~nm}$ & $1 \mathrm{a} 4 \mathrm{~J} / \mathrm{cm}^{2}$ & $1.8 \mathrm{a} 3.8 \mathrm{~J} / \mathrm{cm}^{2}$ \\
Q\&RT - C e P & $632.8 \mathrm{~nm}$ a $660 \mathrm{~nm}$ & $2 \mathrm{a} 3 \mathrm{~J} / \mathrm{cm}^{2}$ & $10 \mathrm{a} 100 \mathrm{~mW}$ \\
Q & $632.8 \mathrm{~nm}$ a $660 \mathrm{~nm}$ & $5 \mathrm{~mW} 35 \mathrm{~J} / \mathrm{cm}^{2}$ \\
\hline
\end{tabular}

\section{Considerações finais}

De acordo com as considerações levantadas neste estudo, pode-se considerar que o uso do laser de baixa intensidade é um método efetivo para a prevenção e o tratamento da mucosite bucal induzida pela terapia antineoplásica. Os resultados dos tratamentos são variáveis, e os diferentes parâmetros dosimétricos não apresentaram diferenças significativas quando da sua utilização. Essas variáveis são dependentes do comprimento de onda, do pulso, da irradiância, da densidade de energia, da potência do aparelho e da duração do tratamento.

Os estudos identificaram que a densidade de energia com tempo adequado entre as exposições parece ser o único parâmetro com efeitos previsíveis no tratamento de feridas em situações clínicas, por acelerar a reparação e diminuição do quadro álgico.

Com base nos ensaios clínicos encontrados nos artigos pesquisados, conclui-se que a terapia com laser de baixa intensidade para prevenção e trata- 
mento da mucosite bucal nos pacientes oncológicos apresenta-se como uma opção viável e que suas propriedades e indicações poderão levar a mudanças significativas na prática clínica.

\section{Abstract}

Objective: the purpose of this review is to present the different randomized clinical protocols using low level laser in the management of oral mucositis, in the last eighteen years of study. Literature review: oral mucositis remains a major problem to be solved in clinical oncology. This cytotoxic effect limits food intake by mouth due to the pain and discomfort in chewing and/or swallowing. Nutritional status may be aggravated contributing to increased morbidity and hospital stay, and providing negative impact on the quality of life of cancer patients, and on their treatment. Disturbances in the integrity and function of the oral cavity are due to the fact that radiotherapy and chemotherapy are not able to destroy tumor cells without damaging normal cells. Numerous treatments are described in literature and there are several methodologies for the treatment of oral mucositis, yet there is no consensus on the best strategy for prevention and therapy. Final considerations: several studies have shown good results with the use of low level laser, which main indications include anti-inflammatory and analgesic effects, and inducing tissue repair. However, there is no definition of a single protocol for this promising resource so far to be applied in the therapy of this pathology.

Keywords: Laser therapy. Low level. Oral mucositis. Chemotherapy. Radiotherapy.

\section{Referências}

1. Wong SF, Wilder-Smith P. Pilot study of laser effects on oral mucositis in patients receiving chemotherapy. Cancer J 2002; 8(3):247-54.

2. Hawkins-Evans D, Abrahamse H. Efficacy of three laser wavelengths for in vitro wound healing. Photodermatol Photoimunol Photomed 2008; 24(4):199-210.

3. Karu T, Pyatibrat L, Kalendo G. Photobiological modulation of cell attachment via cytochrome c oxidase. Photochem Photobiol Sci 2004; 3(2):211-6.

4. Bensadoun RJ, Franquin JC, Ciais G, Darcorurt V, Schubert MM, Viot M, et al. Low-energy He-Ne laser in the prevention of radiation-induced mucositis: a multicenter phase III randomized study in patients with head and neck cancer. Support Care Cancer 1999; 7(4):244-52.

5. Yu W, Naim JO, Lanzafame RJ. The effect of laser irradiation on the release of bFGF from 3 T3 fibroblasts. Photochem Photobiol 1994; 59(2):167-70.

6. Corazza AV, Jorge J, Kurachi C, Bagato VS. Photobiomodulation on the angiogenesis of the skin wounds in rats using different light sources. Photomed Laser Surg 2007; 25(2):102-5

7. Cowen D, Tardieu C, Schubert M, Peterson D, Resbeut M, Faucher C, et al. Low energy Helium-Neon laser in the prevention of oral mucositis in patients undergoing bone marrow transplant: results of a double blind randomized trial. Int J Radiat Oncol Biol Phys 1997; 38(4):697-703.
8. França CM, Nuñez SC, Prates RA, Noborikawa E, Faria MR, Ribeiro MS. Low-intesity red laser on the prevention and treatment of induced-oral mucositis in hamsters. J Photochem Photobiol B 2009; 94(1):25-31.

9. Genot-Klastersky MT, Klastersky J, Awada F, Awada A, Crombez P, Martinez MD, et al. The use of low-energy laser (LEL) for the prevention of chemotherapy-and-or radiotherapy induced oral mucositis in cancer patients: results from two prospective studies. Support Care Cancer 2008; 16(12):1381-7.

10. Kipshidze N, Nikolaychik V, Keelan MH, Shankar IR, Khanna A, Kornowski R, et al. Low-power helium neon laser irradition anhances production of vascular endothelial growth factor and promotes growth of endothelial cells in vitro. Laser Surg Med 2001; 28(4):355-64.

11. Loevschall H, Arenholt-Bindslev D. Effect of low-level diode laser irradiation of human oral mucosa fibroblasts in vitro. Lasers Surg Med 1994; 14(4):347-54.

12. Lima AG, Antequera R, Peres MP, Snitcosky IM, Federico $\mathrm{MH}$, Villar RC. Efficacy of low-level laser therapy and aluminum hydroxide in patients with chemotherapy and radiotherapy-induced oral mucositis. Braz Dent J 2010; 21(3):186-92.

13. Barasch A, Peterson DE, Tanzer JM, D`Ambrosio JA, Nuki $\mathrm{K}$, Schubert MM, et al. Helio-neon laser effects on conditioning-induced oral mucositis in bone marrow transplantation patients. Cancer 1995; 76(12):2550-6.

14. Antunes HS, de Azevedo AM, Bouzas LFS, Adão CAE, Pinheiro CT, Ferreira CG, et al. Low power laser in the prevention of induced oral mucositis in bone marrow transplantation patients: a randomized trial. Blood 2007; 109(5):2250-5.

15. 15. Sandoval RL, Koga DH, Buloto LS, Suzuki R, Dib LL. Management of chemo and radiotherapy induced oral mucositis with low-energy laser: inicial results of A.C. Camargo Hospital. J Appl Oral Sci 2003; 11(4):337-41.

16. Keefe DMK, Shubert MM, Elting IS, Sonis ST, Epstein JB, Raber-Durlacher JE, et al. Update clinical practice guidelines for the treatment of mucositis. Cancer 2007; 109(5):820-31.

17. Nes AG, Posso MBS. Patients with moderate chemotherapy-induced mucositis: pain therapy using low intensity lasers. Int Nurs Rev 2005; 52(1):68-72.

18. Maiya GA, Sagar MS, Fernandes D. Effect of low level helium-neon. (He-Ne) laser therapy in the prevention \& treatment of radiation induced mucositis in head \& neck cancer patients. Indian J Med Res 2006; 124(4):399-402.

19. Rubenstein EB, Peterson DE, Schubert M, Keefe D, McGuire D, Epstein J, et al. Clinical practice guidelines for the prevention and treatment of cancer therapy-induced oral and gastrointestinal mucositis. Cancer 2004; 100(Suppl 9):2026-46.

20. Lopes NNF, Plaper M, Chavantes MC, Lalla RV, Yoshimura EM, Alves MTS. Cyclooxygenase-2 and vascular endothelial factor expression in 5-fluoracil-induced oral mucositis in hamsters: evaluation of two low-intensity laser protocols. Support Care Cancer 2009; 17(11):1409-15.

21. Volpato LER, Volpato MCPF, Castro PHS, Oliveira RC, Machado MAAM. The use of low-level laser therapy in the prevention and treatment of chemotherapy-induced oral mucositis. Applied Cancer Research 2009; 29(3):105-10.

22. Biron P, Sebban C, Gourmet R, Chvetzoff G, Philip I, Blay JY. Research controversies in management of oral mucositis. Support Care Cancer 2000; 8(1):68-71. 
23. Sonis ST, Elting IS, Keefe D, Peterson DE, Schubert M, Hauer J.M. Perspectives on cancer therapy-induced mucosal injury. Cancer 2004; 100(Suppl 9):1995-2025.

24. Arora H, Pai KM, Maiya A, Vidyasagar MS, Rajeev A. Efficacy of He-Ne Laser in the prevention and treatment of radiotherapy-induce oral mucositis in oral cancer patients. Oral Surg Oral Med Oral Pathol Oral Radiol Endod 2008; 105(2):180-6.

25. Jarvis D, MacIver MB, Tanelian DL. Electrophysiologic recording and thermodynamic modeling demonstrate that helium-neon laser irradiation does not affect peripheral Adelta- or C-fiber nociceptors. Pain 1990; 43(2):235-42.

26. Chor A, Torres SR, Maiolino A, Nucci M. Low-power laser to prevent oral mucositis in autologous hematopoietic stem cell transplantation. Eur J Haematol 2010; 84(2):178-9.

27. Jaguar GC, Prado JD, Nishimoto IN, Pinheiro MC, Castro Jr DOde, Cruz Perez D, et al. Low-energy laser therapy for prevention of oral mucositis in hematopoietic stem cell transplantation. Dis Oral 2007; 13(6):538-43.

28. Genot M-T, Klastersky J. Low-level laser for prevention and therapy of oral mucositis induced by chemotherapy or radiotherapy. Curr Opin Oncol 2005; 17(3):236-40.

29. Lucas C, Stanborough RW, Freeman CL, De Haan RJ. Efficacy of low-level laser therapy on wound healing in human subjects: a systematic review. Lasers Med Sci 2000; 15(2):84-93.

30. Bensadoun RJ, Ciais G. Radiation and chemotherapy-induced mucositis in oncology: results of multicenter phase III studies. J Oral Laser Appl 2002; 2(2):115-20.

31. Kuhn A, Porto FA, Miraglia P, Brunetto AL. Low-level infrared laser therapy in chemotherapy-induced mucositis: a randomized placebocontrolled trial in children. J Pediatr Hematol Oncol 2009; 31(1):33-7.

32. Migliorati C, Massumoto C, Eduardo FP, Muller KP, Carrieri T, Haypek $\mathrm{P}$, et al. Low-energy laser therapy in oral mucositis. J Oral Applications 2001; 1(2):97-101.

33. Cruz LB, Ribeiro AS, Rech A, Rosa LGN, Castro Jr CG, Brunetto AL. Influence of low-energy laser in the prevention of oral mucositis in children with cancer receiving chemotherapy. Pediatr Blood Cancer 2007; 48(4):435-40.

34. Abramoff MM, Lopes NN, Lopes LA, Dib LL, Guilherme A, Caran EM, et al. Low-level laser therapy in the prevention and treatment of chemotherapy-induced oral mucositis in young patients. Photomed Laser Surg 2008; 26(4):393-400.

35. Schubert MM, Eduardo PF, Guthrie KA, Franquin JC, Bensadoun RJ, Migliorati CA, et al. A phase III randomized double-blind placebo-controlled clinical trial to determine the efficacy of low level laser therapy for the prevention of oral mucositis in patients undergoing hematopoietic cell transplantation. Support Care Cancer 2007; 15(10):1145-54.

36. Corti L, Chiarion-Sileni V, Aversa S, Ponzoni A, D’Arcais R, Pagnutti S, et al. Treatment of chemotherapy-induced oral mucositis with lightemitting diode. Photomed Laser Surg 2006; 24(2):207-13.

37. Basford JR. Low intensity laser therapy: still not an established clinical tool. Lasers Surg Med 1995; 16(4):331-42.

38. Lino MD, Carvalho FB, Oliveira LR, Magalhães EB, Pinheiro AL, Ramalho LM. Laser phototherapy as a treatment for radiotherapy-induced oral mucositis. Braz Dent J 2011; 22(2):162-5
39. Djavid GE, Emami A, Ataie-Fashtami L, Safaeinodehi Sr, Merikh-Baiat F, Fateh M, et al. Low level laser therapy in management of chemotherapy- induced oral mucositis: prophylaxis or treatment? Lasers Med Sci 2011; 2(1):12-7.

40. Zanin T, Zanin F, Carvalhosa AA, Castro PH, Pacheco MT, Zanin IC, et al. Use of $660 \mathrm{~nm}$ diode laser in the prevention and treatment of human oral mucositis induced by radiotherapy and chemotherapy. Photomed Laser Surg 2010; 28(2):233-7

41. Bjordal JM, Bensadoun RJ, Tunèr J, Frigo L, Gjerde K, Lopes-Martins RA. A systematic review with meta-analysis of the effect of low-level laser therapy (LLLT) in cancer therapy-induced oral mucositis. Support Care Cancer 2011; 19(8):1069-77.

42. Arbabi-Kalati F, Moridi T. Evaluation of the effect of low level laser on prevention of chemotherapy-induced mucositis. Acta Med Iran 2013; 51(3):157-62.

43. Gautam AP, Fernandes DJ, Vidyasagar MS, Maiya AG, Nigudgi S. Effect of low-level laser therapy on patient reported measures of oral mucositis and quality of life in head and neck cancer patients receiving chemoradiotherapy-a randomized controlled trial. Support Care Cancer 2013; 21(5):1421-8.

44. Migliorati C, Hewson I, Lalla RV, Antunes HS, Estilo CL, Hodgson B, et al. Systematic review of laser and other light therapy for the management of oral mucositis in cancer patients. Support Care Cancer 2013; 21(1):333-41.

45. Gautam AP, Fernandes DJ, Vidyasagar MS, Maiya AG, Vadhiraja BM. Low level laser therapy for concurrent chemoradiotherapy induced oral mucositis in head and neck cancer patients - a triple blinded randomized controlled trial. Radiother Oncol 2012; 104(3):349-54.

46. Silva GB, Mendonça EF, Bariani C, Antunes HS, Silva MA. The prevention of induced oral mucositis with low-level laser therapy in bone marrow transplantation patients: a randomized clinical trial. Photomed Laser Surg 2011; 29(1):27-31.

47. Gouvêa de Lima A, Villar RC, de Castro G Jr, Antequera $\mathrm{R}$, Gil E, Rosalmeida MC, et al. Oral mucositis prevention by low-level laser therapy in head-and-neck cancer patients undergoing concurrent chemoradiotherapy: a phase III randomized study. Int J Radiat Oncol Biol Phys 2012; 82(1):270-5.

48. Carvalho PA, Jaguar GC, Pellizzon AC, Prado JD, Lopes RN, Alves FA. Evaluation of low-level laser therapy in the prevention and treatment of radiation-induced mucositis: a double-blind randomized study in head and neck cancer patients. Oral Oncol 2011; 47(12):1176-81.

49. Cauwels RG, Martens LC. Low level laser therapy in oral mucositis: a pilot study. Eur Arch Paediatr Dent 2011; 12(2):118-23.

50. Lubart R, Friedmann H, Peled I, Grossman N. Ligth effect on fibroblast proliferation. Laser therapy 1993; 5:55-7.

\section{Endereço para correspondência:}

Antonio Eugenio Magnabosco Neto Rua Gustavo Capanema, 6, América 89204448 Joinville, SC Fone: (47) 3433-4347 - (47) 9916-0828

E-mail: aemagnabosco@terra.com.br

Recebido: 11/09/2012. Aceito: 06/08/2013. 